\title{
Multimodal Treatment of Thyroid Cancer Bone Metastases Appears to Improve Survival as Compared to I-131 Alone
}

\section{Brian W. Kim}

Division of Endocrinology and Metabolism, Department of Internal Medicine, Rush Medical College, Chicago, Illinois, U.S.A.

Review of: Wu D, Gomes Lina CJ, Moreau SL, Kulkarni K, Zeymo A 2019 Improved survival after multimodal approach with ${ }^{131}$ I treatment in patients with bone metastasis secondary to differentiated thyroid cancer. Thyroid. Epub 2019 Apr 24. PMID: 31017051.

\section{SUMMARY}

\section{Background}

Bone metastases are a defining feature of "advanced" thyroid cancer, being associated with markedly decreased survival. Historical data have suggested that treatment of bone metastases with radioactive iodine (I-131) may improve survival, though the ATA 2015 Guidelines also state that such therapy is "rarely" curative (1-3). Small studies have suggested clinical benefits for a wide range of therapies, including surgical resection, radiation, embolization, cryotherapy, radiofrequency ablation, antiresorptive drugs, and systemic therapies. Some of these convey symptomatic benefits and are thus widely used, but in general the effects of these methods on survival outcomes are not clear, and guidance as to when to employ them is lacking.

In the current study, investigators at a single large institution performed a retrospective analysis to determine whether there was any survival difference between advanced thyroid cancer patients with bone metastases treated only with I-131 and patients who had received $\mathrm{I}-131$ in combination with other therapies (4).

\section{Methods}

This was a retrospective study of patients evaluated between 2001 and 2018 at MedStar Washington Hospital Center. All subjects had differentiated thyroid cancer (DTC), bone metastasis (BM), and at least one I-131 treatment after the diagnosis of BM. Patients treated only with I-131 were compared to patients treated with I-131 and at least one other therapy, including BM surgery, radiofrequency ablation, cryotherapy, arterial embolization, external-beam radiation, CyberKnife treatment, antiresorptive medication, and systemic targeted therapy. The primary end point was all-cause mortality. Overall survival (OS) was defined as the period from initial diagnosis of BM to death. OS was analyzed by Kaplan-Meier survival curves and was compared by log-rank testing between two groups at 1, 2, 3, 5, and 10 years.

\section{Results}

A total of 77 patients met the above criteria and had been followed for up to 41 years. A total of $30 \%$ (23 of 77 ) of patients had received I-131 treatment alone, and the other $70 \%$ (54 of 77) received multimodal therapies. OS was lower at all 
time points for the I-131-only group. For I-131only, the median survival was 3.9 years, and OS $(1,2,3,5$, and 10 years) were $86 \%, 81 \%, 61 \%$, $35 \%$, and $23 \%$. In comparison, those who received a combination of therapies had a longer median survival of 7.7 years, and OS was better $(96 \%$, $92 \%, 86 \%, 69 \%$, and $30 \%)$.

Median survival after BM diagnosis was higher for several subgroups: those whose initial I-131 therapy was within 6 months after thyroidectomy versus later ( 6.5 vs. 0.5 years; $P<0.001$ ); those who received external-beam therapy at some point (7.8 vs. 4.4 years; $\mathrm{P}=0.016$ ); those who received denosumab (7.7 vs. 5.2 years; $P=0.03$ ): and patients whose age was $<55$ years at the time of initial diagnosis or at the time of BM diagnosis as compared with older patients $(P=0.01)$. However, in the multivariate analysis, only the age at initial diagnosis of DTC and timing of initial I-131 therapy within 6 months after thyroidectomy were independent prognostic factors.

\section{Conclusions}

In patients with differentiated thyroid cancer with iodine-avid bone metastases, combination treatment with I-131 and other therapies was associated with improved OS as compared with treatment with I-131 alone.

\section{COMMENTARY}

The results of this retrospective study are consistent with the evolving belief that more aggressive therapy directed against bone metastases is not futile. The finding that combination therapies seem to be associated with improved survival is in general agreement with a number of other small studies. For example, Kato and colleagues reported that complete surgical resection of spinal metastases, when possible, improved both performance status and overall survival as compared with incomplete resection (5). That said, there are few controlled trials looking at any of the individual methods.

Strengths of this study include its being one of the larger studies in the literature with results specific to patients with differentiated thyroid cancer (poorly differentiated and anaplastic thyroid cancer patients were excluded) and the use of OS as an end point.

At the same time, the retrospective nature of the study limits our ability to make specific assumptions as to why the combination therapy group did better; indeed, the reasons why some patients received only I-131 are not apparent. An institution-specific feature that may limit the generalizability of the findings is the use of dosimetrically guided I-131 in patients with bone metastases; this study is perhaps the largest of its kind examining dosimetric I-131 with respect to the use of combination therapies. It is left to future studies to tell us if any particular combination of noniodine methods might be better than individual methods. 


\section{References}

1. Haugen BR, Alexander EK, Bible KC, Doherty GM, Mandel SJ, Nikiforov YE, Pacini F, Randolph GW, Sawka AM, Schlumberger M, et al. 2016 2015 American Thyroid Association management guidelines for adult patients with thyroid nodules and differentiated thyroid cancer: the American Thyroid Association Guidelines Task Force on Thyroid Nodules and Differentiated Thyroid Cancer. Thyroid 26:1-133.

2. Bernier MO, Leenhardt L, Hoang C, Aurengo A, Mary JY, Menegaux F, Enkaoua E, Turpin G, Chiras J, Saillant G, Hejblum G 2001 Survival and therapeutic modalities in patients with bone metastases of differentiated thyroid carcinomas. J Clin Endocrinol Metab 86:1568-1573.

3. Schlumberger M, Challeton C, De Vathaire F, Travagli JP, Gardet P, Lumbroso JD, Francese C, Fontaine F, Ricard M, Parmentier C 1996 Radioactive iodine treatment and external radiotherapy for lung and bone metastases from thyroid carcinoma. J Nucl Med 37:598-605.
4. Wu D, Gomes Lina CJ, Moreau SL, Kulkarni K, Zeymo A 2019 Improved survival after multimodal approach with ${ }^{131}$ I treatment in patients with bone metastasis secondary to differentiated thyroid cancer. Thyroid. Epub 2019 Apr 24.

5. Kato S, Murakami H, Demura S, Fujimaki Y, Yoshioka K, Yokogawa N, Tsuchiya H 2016 The impact of complete surgical resection of spinal metastases on the survival of patients with thyroid cancer. Cancer Med 5:2343-2349. 